\title{
COVID-19 Early Detection Tool for Elder Abuse during Epidemics, Digital Analysis of Color Tone on the Surface of the Skin in Elderly People
}

\author{
Noriko Yamada ${ }^{1}$, Hideki Hyodoh ${ }^{2}$, Tomoko Matsuhashi ${ }^{3} \&$ Shinichi Oikawa $^{3}$ \\ ${ }^{1}$ School of Nursing, Japanese Red Cross Akita Colledge of Nursing, Akita, Japan \\ ${ }^{2}$ School of Medecin, Hokkaido University, Hokkaido, Japan \\ ${ }^{3}$ School of Caring, Japanese Red Cross Colledge of Akita, Akita, Japan \\ Correspondence: Noriko Yamada, Japanese Red Cross Akita Colledge of Nursing, Akita, Japan, 17-3 \\ Nawashirosawa, Kamikitate, Akita, Japan. Tel: 81-18-829-4311.
}

Received: February 11, 2021 Accepted: March 12, 2021 Online Published: March 17, 2021

doi:10.5539/gjhs.v13n5p1 URL: https://doi.org/10.5539/gjhs.v13n5p1

\begin{abstract}
The purpose of this study was to attempt a digital analysis of body color tone of elderly subjects, thus demonstrating that nurses and caregivers can easily and reliably record changes in body color tone.

This cross-sectional study took place between April 1, 2017 and March 31, 2019. A workshop was set up where observers received explanations from researchers on how to use color charts and recording forms. Measurement instruments (digital cameras) were also standardized in this effort. While the elderly subjects targeted by this study suffered from dementia, they were able to converse and understood the purpose of the study, and the study was conducted with their and their families'consent. In addition, after receiving approval from a research ethics examination from an affiliated university, the target facility gaining this consent was subjected to an ethical review, after which we implemented the study in accordance with ethical guidelines for medical research on humans.

Consent was obtained from 30 subjects (20 female $(66.7 \%), 8$ male $(26.7 \%)$ and 2 for which the gender was unknown; average age: 87.8 years (minimum 80 years, maximum 100 years)). We were able to perform digital image analysis of the lesion site and unaffected parts, and present numerical values.

Evaluations by observers were significantly different depending on the individual, and subjectivity greatly influenced comparisons with the color chart based on visual evaluations. It was confirmed that numerical evaluation of images taken in hospitals and nursing homes could also be performed using general-purpose software.
\end{abstract}

Keywords: color chart and scale, digital analysis, elderly, body color tone

\section{Introduction}

\subsection{Introduce the Problem}

In recent years, it is increasingly common to hear about growing populations of elderly people with dementia, and cases of caregiver abuse. We initially attempted a technique involving observing skin discoloration with the naked eye to check for markers of being hit, struck, or stepped on, and logging these findings, but $80 \%$ of participants were unable to accurately perform an observation (Yamada-b, 2020). Therefore, we thought that a tool capable of objectively evaluating and recording the color of the skin on the body surface was needed. We received the consent of the elderly to take photographs and use these in conjunction with reference to their regular nursing records.

With age, the skin of the elderly becomes dry, its elasticity decreases, and various skin complications are more likely to occur. Due to individual differences thickness of fat deposits, original skin color, skin tension or tautness, and moisture content, detecting abnormalities based on skin color alone does not suffice. Generally, nursing observation records include complaints lodged by elderly people as regard bleeding, inflammation, rashes, blisters, and itching and pain.

\subsection{Explore Importance of the Problem}

When reviewing prior literature on the subject, we found many papers discussing skin discoloration in the elderly 
in the context of bedsores. Patients with vertebral injury, reduced motor skills from long periods of convalescence, and those with neurological disorders exhibit reduced activity, causing redness and blisters at sites were body pressure is concentrated at the same position for prolonged periods of time, causing bedsores. The use of bedsore prevention guidelines as a risk assessment tool is currently recommended to clinical practitioners. In prior work (Moore \& Patton, 2019) on the efficacy of said risk assessment tools, the report found that, in a population of 1,487 at risk of bedsores, there was no discernible difference in the extent to which the Braden, Waterlow, and Ramstadius risk assessment scales had on the prevention of bedsores over customary clinical judgment modalities.

Screening patients without clear signs of abuse in medical contexts could aid in the identification of those at risk and reduce their exposure to violence and abuse. In Japan, Mimasaka (2010, 2012a, 2012b, 2018) has attempted to diagnose signs of child abuse based on the color of bruises.

According to literature discussing the United States Preventive Services Task Force's IPV screening methodology and abuse of elderly and vulnerable people (Nelson, Bougatsos, \& Blazina, 2012), the Elder Abuse Suspicion Index (EASI) yields a sensitivity of 9-47 percent and specificity of 75-97 percent based on the number of affirmative replies to questions. However, studies of abuse in the elderly and vulnerable adults remain scarce. Moreover, we did not find any RCT studies (Feltner et al., 2018) on the inadequate care and neglect of the elderly and treatment of abuse. In the authors' own experience, while some subjects paged nurses for help all through the night, still others refrained from seeking aid to an extent that would leave one wondering why they did not seek help sooner.

\subsection{State Hypotheses and Background}

Japan is one of the foremost ageing societies in the world, with three points of interest: 1) there are approximately 4M elderly people with mild cognitive impairment that are at risk of dementia (JCO, 2019); 2) elder abuse is on the rise (JCO, 2017), necessitating screenings for early detection of said incidents; and 3) there is no extant scale used for observation of signs of abuse on the skin. Given the above, the authors sought to develop a method of observation of skin tone changes in elderly populations through the use of color charts and scales.

Seeking to resolve the three points above, we developed an objective observation method based on the use of skin surface scales and attempted to perform digital analysis of the skin tone in elderly subjects, and demonstrate the viability of accurately recording said changes in a manner that is minimally invasive to nurses and caregiving practitioners.

\section{Method}

This was a cross-sectional study performed from April 1, 2017 to March 31, 2019.

\subsection{Test population}

Elderly persons with discoloration of the skin on the surface of the body.

Of the above group, those hospitalized or regularly commuting to a hospital or care facility.

\subsection{Methodology}

\subsubsection{Standardization of Observation Criteria}

Seeking to standardize the observation criteria, to the extent possible, subjects were observed in the same location, at the same time, using the same light source (fluorescent lamps, etc.). Digital cameras were used for daily photographs in order to minimize individual differences that are produced in observations made by the naked eye. The same model of digital camera was used and shooting conditions standardized, with these units then distributed to participating facilities and participants asked to not change the settings.

\subsubsection{Observation by Nurses and Caregivers}

Prior to each photography session, the consent of the subject was obtained, and regions of the skin exhibiting a changed color were photographed.

These photographs were taken in private rooms or treatment areas to ensure privacy. For the first session, the entire body of the subject (front and in profile) was photographed, as well as specific regions alongside which the color chart and scale were placed. For subsequent sessions, the color chart and scale were used on regions exhibiting changed color, with the region photographed such that its midpoint was in the center of the frame. The image data was then cross-checked with patient logs. In the event of signs of swelling or inflammatory exacerbation, the matter was reported immediately to the attending physician. 


\subsubsection{Digital Analysis of Color Abnormalities on the Body Surface}

Utilizing image analysis software, we compared digital images of the surface of the skin in which abnormalities were observed with the naked eye with a control set of images showing the skin in normal conditions.

Photographic equipment: Canon IXY190

Photography criteria: no strobes, photographed simultaneously with color chart and scale

\subsubsection{Image Analysis Methodology}

2.2.4.1 Naked eye evaluation: abnormal and normal areas are examined and their relative color and size compared.

2.2.4.2 Digital photographic image analysis: the photos taken were verified using Adobe Photoshop CC (2015 release) as follows:

i. Observe the JPEG image data with the naked eye, and select the entirety, and center of, regions deemed to be of abnormal color using the Lasso tool.

ii. Select Window -> Histogram and record the average, standard deviation, median, and pixel values displayed for $\mathrm{RGB} / \mathrm{red} /$ green/blue/luminance/color.

iii. Perform comparison of skin tone in areas subject to injury with normal skin tone. Moreover, for those cases in which observation of tonal changes over time was possible, we also examined the characteristics of said change.

\subsubsection{Ethical Considerations}

We discussed the purposes of this study and its ethical considerations with the directors of hospitals and care facilities and obtained their consent. We then held sessions with nurses and caregivers at the wards of said sites to discuss the research and obtain their cooperation, both orally and in writing. We requested the observation of approximately twenty elderly people at each facility. Those subjects capable of understanding the purpose of the research (including those with dementia, provided they could communicate and comprehend the project) were eligible, and consent of the individuals and their families was obtained. A copy of a consent form was retained by the subject and researcher, respectively, with subjects given contact details for the researcher and a consent withdrawal request form, giving them the right to revoke their participation at any time and make contact as necessary.

Posters indicating the purpose and scope of the research and explaining the use of cameras were posted so as to be visible to subjects and their families, and oral and written consent was specifically obtained from those subjects requiring observation. Daily photographs were performed by a nurse or practitioner with the consent of the subject. The research was conducted with The Ethnical Guidelines for Nursing Research and the approval of the Research Ethics Review Committees of the Japanese Red Cross Akita College of Nursing and the Japanese Red Cross Junior College of Akita.

\section{Results}

\subsection{Subject Attributes and Naked Eye Observation (Table 1)}

We obtained the assistance of three hospitals and five caregiving facilities. We analyzed thirty cases for subjects from whom consent was obtained. (20 females, $66.7 \% ; 8$ males, $26.7 \% ; 2$ unspecified. Average age: 87.8)

Among their medical histories were: Alzheimer's disease (6 subjects, 20.0\%); cerebral infarction ( 3 subjects, $10.0 \%$ ); and use of anticoagulants or antiplatelets pursuant to administration of drugs with a tendency to cause bleeding (4 subjects, $13.3 \%$ ).

We prepared two forms: one used for initial observations, and one for daily observations. Approximately eighty percent of remarks regarding skin tone changes and overall bodily condition involved statements on nutrition, physique, medicine being taken under treatment, and currently administered treatments. We also asked observers to record on a body map where skin tone changes were observed, but only about twenty percent considered the size, region, or type of change, and several miss-mapped the left and right sides. The regions in which skin tone changes were observed were: upper body $(19,63 \%)$; lower body $(18 ; 60.0 \%)$; and head $(4 ; 13.3 \%)$. 
Table 1. Attributes and physical conditions of the subject

\begin{tabular}{|c|c|c|c|}
\hline & Item & $\mathbf{n}$ & $\mathrm{N}=\mathbf{3 0}(\%)$ \\
\hline \multirow{4}{*}{ Gender } & Male & 8 & $(26.7)$ \\
\hline & Female & 20 & $(66.7)$ \\
\hline & Unknown & 2 & $(6.6)$ \\
\hline & Average age (80-100 y.o.) & 87.8 & \\
\hline \multirow{5}{*}{ Medical history } & Alzheimer dementia & 6 & $(20.0)$ \\
\hline & Cerebral infarction & 3 & $(10.0)$ \\
\hline & Cancer & 1 & $(3.3)$ \\
\hline & None & 18 & $(60.0)$ \\
\hline & Unknown & 2 & $(6.7)$ \\
\hline \multirow{4}{*}{ Medicine taken } & Anticoagulant & 4 & (13.3) \\
\hline & Antiplatelet agent & 4 & $(13.3)$ \\
\hline & ADP inhibitor & 1 & $(3.3)$ \\
\hline & None & 21 & $(70.1)$ \\
\hline \multirow{22}{*}{ Region (can overlap) } & Head & 4 & $(13.3)$ \\
\hline & Breakdown: head & 1 & $(3.3)$ \\
\hline & face & 3 & $(10.0)$ \\
\hline & neck & 0 & $(0.0)$ \\
\hline & Upper extremity & 19 & $(63.3)$ \\
\hline & Breakdown: upper arm & 2 & $(6.7)$ \\
\hline & forearm & 5 & $(16.7)$ \\
\hline & supraclavicular fossa & 1 & $(3.3)$ \\
\hline & shoulder & 1 & $(3.3)$ \\
\hline & cubital region & 1 & $(3.3)$ \\
\hline & wrist & 4 & $(13.3)$ \\
\hline & dorsum of hand & 4 & $(13.3)$ \\
\hline & finger & 1 & $(3.3)$ \\
\hline & Lower extremity & 18 & $(60.0)$ \\
\hline & Breakdown: sacral region & 5 & $(16.7)$ \\
\hline & lower limbs & 2 & $(6.7)$ \\
\hline & knee & 3 & $(10.0)$ \\
\hline & calcaneal region & 2 & $(6.7)$ \\
\hline & ankle & 1 & $(3.3)$ \\
\hline & toe & 3 & $(10.0)$ \\
\hline & dorsalis pedis & 2 & $(6.7)$ \\
\hline & No answer/mistake & 2 & $(6.7)$ \\
\hline
\end{tabular}

Table 2 discusses the size and color observations made per the analog color chart and scale provided. We originally intended for the observation process to last for about ten days, but at the shortest, participants recorded their findings for a period of twenty days, and at the longest, or a period of over two months.

As seen in Table 2, surface skin discolorations observed with the naked eye expanded for about a week from the time of discovery, and then gradually changed in color, with the extent of discoloration subsiding. 
Table 2. Naked eye observation of skin discoloration on body surface using color charts

\begin{tabular}{|c|c|c|c|c|c|c|}
\hline Age/gender & \multicolumn{2}{|c|}{82 y.o. male } & \multicolumn{2}{|c|}{85 y.o. male } & \multicolumn{2}{|l|}{86 y.o. male } \\
\hline Medical history & \multicolumn{2}{|l|}{ None } & \multicolumn{2}{|c|}{ Kidney failure } & \multicolumn{2}{|c|}{ Heart failure (Heart pacemaker) } \\
\hline Medicine taken & \multicolumn{2}{|l|}{ None } & \multicolumn{2}{|c|}{ Anticoagulant } & \multicolumn{2}{|l|}{ Anticoagulant } \\
\hline Cause & \multicolumn{2}{|c|}{ Unknown cause } & \multicolumn{2}{|l|}{ Dialysis } & \multicolumn{2}{|l|}{ Fell at night } \\
\hline \multirow[t]{2}{*}{ Discolored area } & \multicolumn{2}{|c|}{ radial side of forearm } & \multicolumn{2}{|c|}{ medial aspect of the arm } & \multicolumn{2}{|c|}{ forehead and both orbital regions } \\
\hline & Size $(\mathrm{cm})$ & Color code & Size $(\mathrm{cm})$ & Color code & Size $(\mathrm{cm})$ & Color code \\
\hline 0日 & $8.5 \times 2.5$ & 8 & $13.5 \times 7.0$ & 18 & $5.5 \times 4.0$ (forehead) & 19 \\
\hline 1 day later & $8.5 \times 3.0$ & 9 & & & $5.5 \times 4.0($ forehead $)$ & 19 \\
\hline 2 days later & & & & & $5.5 \times 4.5$ & 19 \\
\hline 3 days later & $9.0 \times 5.0$ & 16 & & & & \\
\hline 4 days later & $9.0 \times 5.0$ & 17 & & & & \\
\hline 5 days later & $8.0 \times 4.0$ & 17 & & & & \\
\hline 7 days later & & & $22.0 \times 9.5$ & 17 & & \\
\hline 9 days later & $5.5 \times 4.0$ & 17 & & & $6.5 \times 5.5($ forehead $)$ & 18 \\
\hline 10 days later & $5.0 \times 4.0$ & 17 & & & & \\
\hline 11 days later & $4.5 \times 3.0$ & 18 & & & & \\
\hline 12 days later & $4.0 \times 3.5$ & 23 & & & & \\
\hline 13 days later & $4.0 \times 3.5$ & 23 & & & & \\
\hline 14 days later & $3.0 \times 3.5$ & 24 & $24.0 \times 11.5$ & 17 & & \\
\hline 16 days later & $3.0 \times 2.5$ & 24 & & & & \\
\hline 17 days later & $2.0 \times 1.0$ & 1 & & & & \\
\hline 18 days later & $1.0 \times 1.0$ & 1 & & & & \\
\hline 19 days later & $1.0 \times 1.0$ & 1 & & & & \\
\hline 21 days later & & & $23.0 \times 10.5$ & 16 & & \\
\hline 23 days later & & & & & $7.0 \times 14.5$ (orbit) & 17 \\
\hline 28 days later & & & $22.0 \times 9.5$ & 16 & & \\
\hline 30 days later & & & & & $8.0 \times 14.5$ (orbit) & 17 \\
\hline 35 days later & & & $22.0 \times 9.5$ & 16 & & \\
\hline 38 days later & & & & & $7.0 \times 14.5$ (orbit) & 16 \\
\hline 42 days later & & & $21.0 \times 9.0$ & 16 & & \\
\hline 45 days later & & & & & $7.0 \times 13.5$ (orbit) & 14 \\
\hline 49 days later & & & $21.0 \times 8.5$ & 16 & & \\
\hline 52 days later & & & & & $4.0 \times 13.0$ (orbit) & 14 \\
\hline 56 days later & & & $21.0 \times 9.0$ & 16 & & \\
\hline 59 days later & & & & & $3.5 \times 13.0$ (orbit) & 13 \\
\hline 63 days later & & & $21.0 \times 8.5$ & 16 & & \\
\hline
\end{tabular}

Color codes: 2-7 green shades 8-14 blue shades, 15-17 purple shades, 18-22 red shades, 23, 24, 1 orange to yellow.

Note. Table 2 repeated measure ANOVA was performed for statistical analysis. Statistically, there is no significant difference in the change over time, but it can be seen that the difference in color tone between the lesion and the normal part decreases with the passage of time. 


\subsection{Digital Image Analysis}

\subsubsection{Digital Image Analysis Methodology Explained}

\subsubsection{Observation with the Naked Eye}

Differences in color tone rendered onscreen allow for distinguishing between lesions and normal regions. The color ranged from dark purple-red to purple-red, with some areas exhibiting a blue-purple and yellow-brown tone. No additional change in the above was observed over time.

\subsubsection{Digital Image Analysis}

Digital analysis of normal regions and lesions was viable, as was numerical quantification.

\subsubsection{Digital Analysis}

Collected image data: 1,878 images.

\subsubsection{Observation with the Naked Eye}

Normal skin tone was the expected skin color when observed with the naked eye, but the injured area ranged from purple-red to dark purple-blue, also developing a yellow tone and some areas becoming lighter in color over time. While regions exhibiting abnormal tone gradually decreased and abated over time, in some cases, there were regions that exhibited no marked change during the observation period. There appears to be considerable individual difference and difference from region to region, and the health of the subject is heavily implicated in the formation of objective assessments.

\subsubsection{Digital Imaging}

i. Comparison of normal and abnormal regions

We compared the RGB, red, green, blue, brightness, and color values of the subject's normal surface skin color and that in discolored regions. There was a statistically significant difference between the discolored region and the normal region. (Risk factor $\mathrm{p}<.05$, paired t-test)

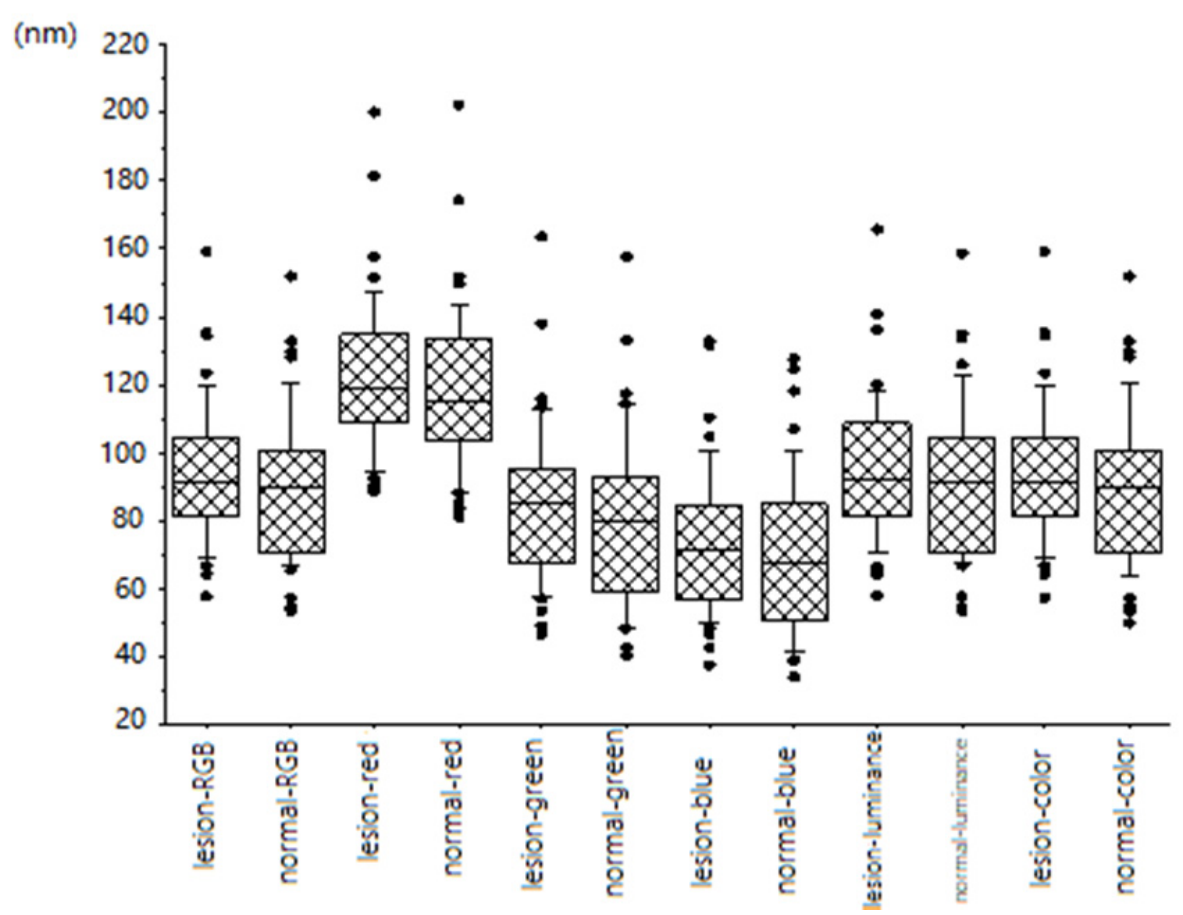

Figure 1. Digital analysis of difference between normal skin color and sites of skin discoloration

Note. This figure is an analysis of color components. RGB is a comparison of all tones, red, green blue, brightness, and color. The paired t-test is used for statistical analysis.

ii. Observation of tonal changes over time (Figure 2)

No statistically significant difference (using the Repeated Measures ANOVA modality) was observed in terms of 
changes over time between lesions and normal regions.

Individual differences in evaluations made by observers were considerable, with naked eye observations against the color chart being influenced by subjective opinions. The research found it viable to numerically quantity the results of photographs taken at hospitals and care facilities and use general-purpose software for image analysis.

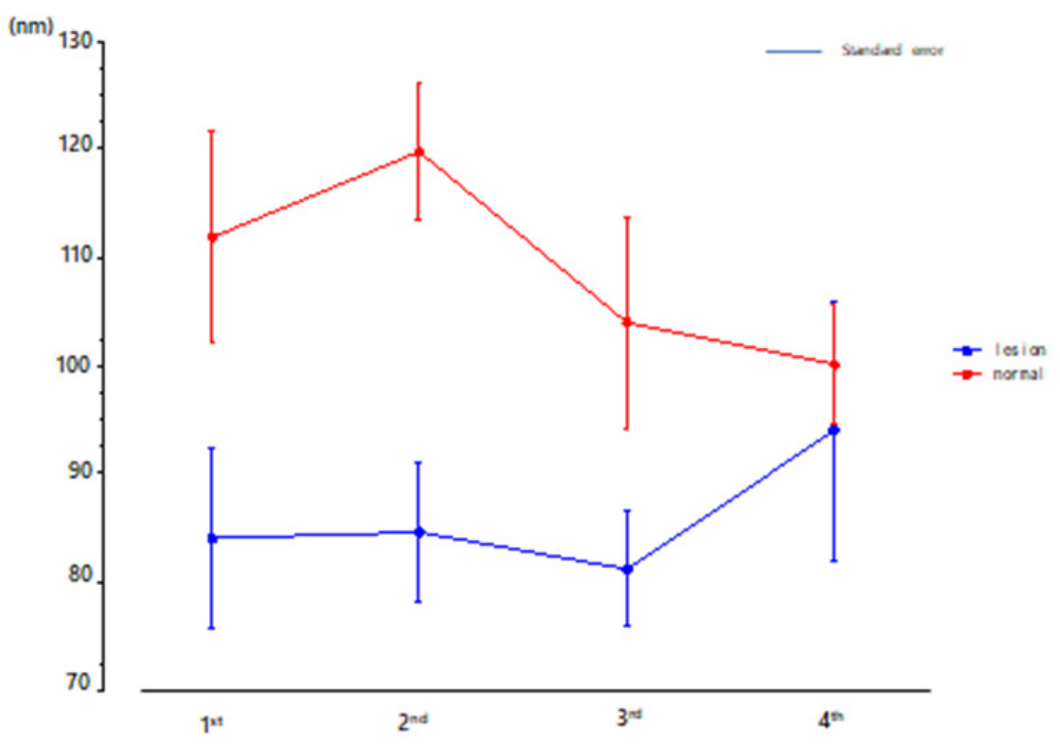

Figure 2. Changes in body surface skin discoloration and normal skin color over time

Note. Figure 2 confirmed the $\mathrm{F}$ value and $\mathrm{p}$ value using multivariate analysis of variance. The upper polygonal line shows the change in color tone of the normal part, and the lower polygonal line shows the change in color tone of the lesion part.

\section{Discussion}

For this study, we observed the surface of the skin in an elderly population using color charts and scales, using these records to develop a tool enabling the early detection of the need for intervention.

\subsection{Objective Evaluation of Skin on the Body Surface}

We attempted to perform a digital analysis of the skin tone on the surface of the body of elderly subjects, and compare the resulting RGB, red, green, blue, luminance, and color values of normal (control) skin, and that exhibiting changes or abnormalities, with each other. A significant difference was found between the normal and changed regions. We demonstrated the viability of a method using digital cameras to record photographs and allow nurses and caregivers to easily log changes in patients' skin tone. In addition, over $80 \%$ of remarks made concerned the subject's medical history, current treatment and medicine, the skin surface, and condition of the nails. However, observers appeared to find it difficult to accurately describe in writing the size and location of abnormalities.

When performing digital image analysis, we attempted to consistently handle differences in image size using the scale simultaneously photographed. However, due to the photographing angle, some lesions required evaluation in the absence of such confirmation. Ensuring consistent distance from the light source proved difficult, and some lesions exhibiting differences in brightness were observed. We interpreted this to be an error in the imaging process. It was difficult to make a determination on some images because the angle and size were not uniform when photographing the surface of the discolored skin. To compensate for this, in addition to digital cameras, laptop computers with built-in webcams were installed with the color chart and scale software as an additional means of logging skin tone changes. This allowed for logging and retaining private information separately from patients' actual medical records. Moreover, it enabled nurses and caregivers to save time when logging changes in the size and tone of regions under observation and accurately record changes in skin tone.

\subsection{Viability of This Scale}

Upon beginning our research, we found it necessary to obtain a tool for the early detection of abuse in elderly and 
disabled populations (Yamada, 2016; Yamada, 2020a). However, prior work by Nelson et al. (2012) and Feltner et al. (2018) suggested that there was no objective scale extant. Klasinc et al. (2017) has utilized MRI to analyze wounds on the region cervicalis posterior and observe bleeding in the sternocleidomastoid muscle; Kondou (2017) demonstrated the efficacy of utilizing an immunohistochemical approach to AQP3 in skin in the neck region following ligature strangulation to arrive at forensic conclusions. Ordinarily, detection and diagnosis of bruises is performed by the naked eye under a light source. In some cases, bruises in victims of abuse cannot be readily observed due to factors such as skin color and age. Prior work has found that using an alternate light source is five times more effective at detecting a range of bruises in victims, regardless of skin tone, than white light (Scafide et al., 2020). By contrast, alternate light sources can be used to detect bruises, but other skin lesions (scars, hyperpigmentation, etc.) may appear identical when using this technique, so Scafide (2020) advises against using it for the diagnosis of bruises.

In engaging with elderly populations, we found that some subjects are under considerable duress, but the act of seeking aid is perceived as humiliating and as potentially threatening to their way of life. This is not because abuse victims lack the ability to seek aid as such, but because the circumstances in which they find themselves are quite harsh or extreme; there appear to be complex sets of circumstances preventing them from making cries for help. How to properly interpret cries for help from those in extremely trying situations is a support-side issue; it is not the victims' inability to seek aid that is the problem, but sundry issues such as caregivers inability to detect these cries for help, or such observations and care being eschewed out of busy schedules or cost concerns. Use of the color charts and scale for observation of the skin surface hinges on both digital quantification of the results and of an "analog" approach involving empathetic dialogue with elderly subjects, and using these in conjunction is more effective.

\subsection{Future Issues}

As seen in the case of the eighty-five year-old in Table 2, it takes considerable time for subcutaneous tissue damage in the elderly to heal, so there were some pathologies in which no change was perceived with the naked eye during the observational period. Differences in the size of recorded photographs were compensated for by using the scale photographed at the same time as the content therein. We also observed some photographs in which the angle at which they were taken impeded forming a definitive conclusion, so the methodology observers' use for taking the photos is one possible issue to consistent implementation. Therefore, utilizing this color chart and scale requires first undergoing training in correct photography methods.

Moreover, digital analysis of changes in a given lesion revealed quantitative changes over time, but there were many lesions the change of which could not be distinguished with the naked eye alone. One current flaw of this model is its inability to account for this discrepancy between the naked eye evaluation and digital analysis, and we consider it a future issue for improvement.

\section{Conclusions}

This pandemic has changed our lives. At work and at home, they were divided into strong and weak, those with control and those who had no choice but to obey. Many older people fall into the latter category due to illness and diminished physical fitness. A feature of this study is that nurses and caregivers collaborated to observe the skin of the elderly and found tools that help early detection of abuse and self-neglect.

This research revealed the following:

1) Digital images allow for analysis and comparison of lesions and normal (control) areas. Moreover, general-purpose software allows for numerically quantifying and evaluating said images.

2) Differences in the size of recorded photographs can be compensated for by using a scale.

Photographed at the same time as the content therein. Utilizing this scale requires undertaking training in correct photography methods.

\section{Acknowledgements}

We wish to sincerely express our gratitude to the elderly people (and their families) who participated in this study, and the nursing staff who took time out of their busy schedules to record observational logs.

\section{Competing Interests Statement}

The authors declare that there are no competing or potential conflicts of interest.

\section{References}

Feltner, C., Wallace, I., Berkman, N., Kistler, C., Middleton, J. C., .. \& Jonas, D. E. (2018). Screening for Intimate 
Partner Violence, Elder Abuse, and Abuse of Vulnerable Adults. An Evidence Review for the U.S.,21 Nov 2018. https://doi.org/10.1001/jama.2018.13212

Japan Cabinet Office. (2017). 2017 White Paper on Elderly People in Society, Section 2: Posture of elderly people and current conditions and trends in their living contexts. Retrieved from https://www8.cao.go.jp/kourei/whitepaper/w-2017/html/gaiyou/s1_2_3.html

Japan Cabinet Office. (2019). 2019 White Paper on Elderly People in Society. Current conditions surrounding an ageing society and future outlook. Retrieved from https://www8.cao.go.jp/kourei/whitepaper/w2019/html/zenbun/s1_1_1.html

Klasinc, I., Ogris, K., Widek T., et al. (2017). Detection of internal soft tissue injuries of the neck using MRI in victims of survived strangulation without any external findings. 10th international symposium advances in legal medicine combined with the 96th annual conference German society of legal medicine, Germany, 27(4) 353. https://doi.org/10.1007/s00194-017-0182-x

Kondou, T., Ishida, Y., Nosaka, M., Yamamoto, H., \& Hashizume, Y. (2017). Forensic diagnosis of compression based on expression of aquaporin-3 in human neck skin. 27(4), 333.

Mimasaka, S., Ohtani, M., \& Kuroda, N. (2010). Spectrophotometric Evaluation of the Age of Bruises in Children: Measuring Changes in Bruise Color as an Indicator of Child Physical Abuse. The Tohoku Journal of Experimental Medicine, 220(2), 171-175. https://doi.org/10.1620/tjem.220.171

Mimasaka, S., Oshima, T., \& Ohtani, M. (2012). Characterization of bruises using ultrasonography for potential application in diagnosis of child abuse. Legal Medicine. 14(pp. 6-10). https://doi.org/10.1016/j.legalmed.2011.09.007

Mimasaka, S. (2012). Objective methodology for evaluation of bruises in abused children. Forensic Pathology. $8(1), 17-21$.

Mimasaka, S., Oshima, T., \& Ohtani, M. (2018). Visualization of old bruises in children: Use of violet light to

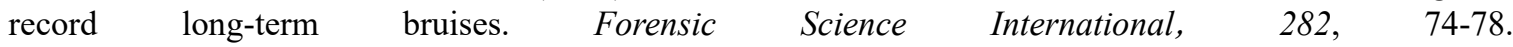
https://doi.org/10.1016/j.forsciint.2017.11.015

Moore, Z., \& Patton, D. (2019). Risk assessment tools for the prevention of pressure ulcers. Cochrane Systematic Review. https://doi.org/10.1002/14651858.CD006471.pub4

Nelson, H. D., Bougatsos, C., \& Blazina, I. (2012). Screening Women for Intimate Partner Violence and Elderly and Vulnerable Adults for Abuse. Agency for Healthcare Research and Quality (US), Rockville (MD), 08 Jun 2012. PMID: 22675737

Scafide, K. N., Sheridan, D. J., Downing, N. R., \& Hayat, M. J. (2020). Detection of Inflicted Bruises by Alternate Light: Results of a Randomized Controlled Trial. Journal of Forensic Sciences. https://doi.org/10.1111/1556-4029.14294

Yamada, N. (2016). Insights on methods of observation of signs of abuse on the skin of the elderly as an early-stage marker for abuse. Journal of Japan Association of Forensic Nursing, 2(2), 49-56.

Yamada, N. (2020a). Actual condition and prospect of elderly people by body surface inspection tool. The 6th International Nursing Research Conference of World Academy of Nursing Science, 1-282.

Yamada, N., Hyodoh, H., Matsuhashi, T., \& Oikawa, S. (2020b). Observation of bodily surface of elderly people utilizing color charts. Journal of Japan Association of Forensic Nursing, 7(2), Issue currently being printed.

Webster, J., Coleman, K., Mudge, A., Marquart, L., Gardner, G., \& Stankiewicz, M. (2011) Pressure ulcers: effectiveness of risk-assessment tools. A randomized controlled trial (the ULCER trial). BMJ Quality and Safety, 20(4), 297-306. https://doi.org/10.1136/bmjqs.2010.043109

\section{Copyrights}

Copyright for this article is retained by the author(s), with first publication rights granted to the journal.

This is an open-access article distributed under the terms and conditions of the Creative Commons Attribution license (http://creativecommons.org/licenses/by/4.0/). 